\title{
Sanificazione e disinfezione: questioni di igiene ai nostri giorni
} Miriam Di Carlo

PUBBLICATO: 26 GENNAIO 2021

\section{Quesito:}

Alcuni lettori ci chiedono quale sia la differenza di significato tra sanificare e sanitizzare (e dunque tra sanificazione e sanitizzazione) e quale forma sia da privilegiare per indicare le operazioni di detersione; altri ci chiedono se sia da preferire disinfezione a disinfettazione e disinfestazione.

\section{Sanificazione e disinfezione: questioni di igiene ai nostri giorni}

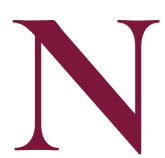

egli ultimi mesi sono state oggetto di discussione, analisi e perfino regolamentazione alcune delle nostre quotidiane abitudini di igiene personale e domestica. Lincertezza circa le modalità di trasmissione del nuovo coronavirus ha portato gli esperti di virologia e malattie infettive (e di conseguenza anche le autorità istituzionali) a mettere in atto unopera di sensibilizzazione circa una più prudente profilassi per scongiurare il contagio anche attraverso la mediazione delle superfici degli oggetti e della densità dell'aria. Le incertezze sulle modalità di trasmissione del virus sono tante e tali da indurci ad adottare sempre e comunque le nuove strategie di igiene, ormai codificate e ritualizzate sia presso ogni singolo individuo (dall'uso di gel igienizzanti alla detersione attenta e accurata delle mani e delle parti maggiormente esposte a un eventuale contagio), sia presso gli ambienti domestici e non (le superfici e i sistemi di aerazione di ospedali, ristoranti, negozi, ecc.). In questo contesto è cresciuto l'uso di verbi come sanificare e sanitizzare, disinfettare e disinfestare e dei relativi sostantivi sanificazione, sanitizzazione, disinfezione, disinfettazione e disinfestazione e ciò fa nascere legittimi dubbi sul loro utilizzo e sui diversi e specifici significati dei vari termini.

Partiamo dai verbi sanificare e sanitizzare, che hanno entrambi come base etimologica l'aggettivo sano (dal latino sanus 'immune da malattie, intatto'). Sanificare aggiunge all'aggettivo sano il suffisso -ificare, tratto dal tema dei composti latini di facēre 'fare' e ha riscontri antichi (il GRADIT e lo Zingarelli 202I segnalano come prima attestazione il I305-6). Fino al XIX secolo il verbo aveva prevalentemente il significato di 'risanare, guarire' non solo il corpo ma anche l'anima, mentre il significato che oggi risulta maggiormente diffuso proviene dal suo derivato sanificazione il cui significato si è specializzato indicando in campo industriale il 'processo atto a rendere gli impianti industriali igienicamente idonei alla produzione di alimenti' e anche il 'trattamento a cui viene sottoposto un alimento per ridurne in misura sufficiente la carica microbica' (GDLI). Dunque dal verbo sanificare (che non aveva in partenza il significato che oggi analizziamo) si è passati al sostantivo sanificazione (che si è specializzato nel significato relativo all'igiene profonda mirata all'eliminazione di agenti patogeni), il quale ha arricchito di una nuova accezione il verbo di partenza: sanificare oggi indica anche 'sottoporre a sanificazione' (GRADIT).

Sanitizzare è invece una parola di più recente formazione: stando al GRADIT risalirebbe al ig83 e avrebbe sempre come base etimologica l'aggettivo sano, mediato questa volta dal sostantivo sanitá con l'aggiunta del suffisso -izzare. I vari dizionari fanno riferimento al verbo inglese to sanitize, che ha senz'altro influito sulla formazione del verbo e sulla sua diffusione, la quale risulta, tuttavia, abbastanza contenuta rispetto al concorrente sanificare. Da sanitizzare è derivato il sostantivo 
sanitizzazione, che il GRADIT riconduce a un sostantivo inglese (sanitization), che però non è registrato nell'OED e conta poche e sporadiche occorrenze nelle pagine in lingua inglese di Google.

Sanificazione ha subito un'evoluzione semantica e a questo proposito non sono da trascurare le primissime occorrenze sui quotidiani nazionali (risalenti alla fine del XIX secolo) in cui il termine contempla il significato di 'purificazione' soprattutto dell'aria, applicata ai processi di bonifica dei terreni:

Pare inoltre che [...] la Società suddetta abbia divisato di costruire una piccola borgata sul centro della terra acquistata, il che oltre di tornare utile alla Società istessa, verrà ad abituare i contadini ad abitare sopra ai terreni che devono lavorare, e così incominciandosi la colonizzazione del piccolo Tavoliere, come chiamasi il territorio brindisino, progredirà in breve l'agricoltura e, cosa al pari interessante, ne varrà la sanificazione dell'aria e la scomparsa delle paludi. ([s.f.], Corriere del Mattino, "la Stampa", 8/5/187i, p. 3)

Confrontando i vari dizionari si nota unulteriore evoluzione di significato: infatti il GDLI e il GRADIT fanno riferimento al solo campo specifico dell'alimentazione industriale mentre lo Zingarelli 202I parla di processi igienici applicabili in qualsiasi campo e a qualsiasi tipologia di ambiente, mirati specificatamente all'eliminazione di agenti patogeni. Le prime occorrenze nell'archivio del "Corriere della Sera" (che risalgono agli anni Settanta del secolo scorso) confermano che sanificazione perteneva strettamente all'ambito industriale e in particolare alimentare:

Nei giorni scorsi ci siamo recati al «Pulisan», la ${ }^{a}$ Mostra Convegno dei prodotti e tecniche della pulizia industriale e della sanificazione, svoltasi nel quartiere della Fiera di Milano. ([s.f.], L’impresa di pulizia oggi è una macchina, "Corriere della Sera", I5/1I/1970, p. 4)

Giovedi prossimo, alla Fiera di Milano si inaugurerà il secondo «Pulisan - Mostra convegno dei prodotti e delle tecniche della pulizia industriale e della sanificazione». [...] Nell'ambito della mostra si svolgerà anche il convegno imperniato su la «Giornata della sanificazione nell'industria alimentare». ([s.f.], In mostra alla Fiera i servizi di pulizia, "Corriere milanese", II-I2/Io/I97I, p. 5)

L'ampliamento di significato registrato per sanificazione (non più relegato al solo campo alimentare) trova in parte una giustificazione nella diffusione concomitante di sanitizzare-sanitizzazione su influsso della semantica inglese: to sanitize significa appunto 'to make sanitary, to disinfect' ['rendere sano, disinfettare'] dove sanitary 'Of or pertaining to the conditions affecting health, esp. with reference to cleanliness and precautions against infection and other deleterious influences' ['delle o pertinente alle condizioni che incidono sulla salute, specialmente con riferimento alla pulizia e alle precauzioni contro le infezioni o altre influenze dannose'].

Riassumendo, nel lessico italiano compare prima il verbo sanificare, attestato già dal XIV secolo, ma con significato differente rispetto a quello attuale. Da sanificare deriva il sostantivo sanificazione, con cui si indicano i processi legati prima alla purificazione dell'aria, poi alla sola igiene industriale (e alimentare) e infine a qualsiasi tipo di pratica vòlta all'eliminazione di agenti patogeni (a partire dall'ambito ospedaliero). Sanificare si specializza in una ulteriore fase nel significato di 'sottoporre a sanificazione'.

Parallelamente, dagli anni Ottanta compare il verbo sanitizzare (da cui deriva sanitizzazione), formato per influsso dell'inglese to sanitize, che ha il significato, non strettamente legato all'ambito industriale ma applicabile anche alle attività domestiche e individuali, di 'sottoporre a sanificazione'. Ad oggi sanificare e sanitizzare, sanificazione e sanitizzazione vengono considerati sinonimi da alcuni dizionari (GDLI, GRADIT e Zingarelli 202I ad esempio) e come tali vengono usati nella maggior parte delle 
occorrenze; le formazioni più antiche, sanificare e sanificazione, risultano più diffuse rispetto alle forme concorrenti:

\begin{tabular}{|c|c|c|c|c|c|}
\hline ricerche 11/9/2020 & sanificare & sanificato/i & sanificata/e & sanifieszione & sanificante \\
\hline $\begin{array}{l}\text { Google } \\
\text { (pagine in italiano) }\end{array}$ & $892.000 \mathrm{r}$ & $\begin{array}{l}595.000 \mathrm{r} \\
533.000 \mathrm{r}\end{array}$ & $\begin{array}{l}170.000 \mathrm{r} \\
325.000 \mathrm{r}\end{array}$ & $7.000 .000 \mathrm{r}$ & $\begin{array}{c}327.000 \mathrm{r} \\
\text { ["gel s." ]" } 73.100 \mathrm{r}\end{array}$ \\
\hline $\begin{array}{l}\text { Repubblica } \\
\text { (I attestazione) }\end{array}$ & 470 r. $(26 / 9 / 2006)$ & $\begin{array}{l}212 \text { r. }(6 / 7 / 2000) \\
272 \text { r. }(1 / 5 / 2004)\end{array}$ & $\begin{array}{l}113 \text { r. }(5 / 8 / 2000) \\
144 \text { r. (18/5/2002) }\end{array}$ & 2.165 r. (26/8/1994) & $\begin{array}{c}50 \text { r. }(14 / 2 / 2020) \\
\text { ["gel s."]; } 19 \text { r. } \\
(10 / 3 / 2020)\end{array}$ \\
\hline ricerche $11 / 9 / 2020$ & sanitizzare & sanitizzato/ & sanitizzata/e & sanitizraxione & sanitizxante \\
\hline $\begin{array}{l}\text { Google } \\
\text { (pagine in italiano) }\end{array}$ & $27.900 \mathrm{r}$ & $\begin{array}{l}21.200 \mathrm{r} \\
11.400 \mathrm{r}\end{array}$ & $\begin{array}{l}14.700 \mathrm{r} \\
6.330 \mathrm{r}\end{array}$ & $77.800 \mathrm{r}$ & $\begin{array}{c}63.000 \mathrm{r} . \\
\text { ["gel s." ]" } 4.460 \mathrm{r} .\end{array}$ \\
\hline $\begin{array}{l}\text { Repubblica } \\
\text { (I attestazione) }\end{array}$ & 1 r. $(24 / 3 / 2020)$ & - & 2 r. $(24 / 3 / 2020)$ & 7 r. $(30 / 3 / 1998)$ & $\begin{array}{c}5 \text { r. }(23 / 7 / 2013) \\
\text { ["gel s."]; } 1 \mathrm{r} \text {. } \\
(27 / 3 / 2020)\end{array}$ \\
\hline
\end{tabular}

La tabella riporta non solo i dati relativi alle occorrenze nelle pagine in italiano di Google, ma anche le attestazioni nell'archivio della "Repubblica" (che parte dal 1984). Per quanto riguarda sanitizzare, la prima attestazione del verbo (e del participio femminile sanitizzata) risale a marzo del 2020, mese in cui è esplosa l'epidemia con la conseguente adozione della severa profilassi. Il participio presente rivela un incremento d'uso legato alla pandemia: sulla "Repubblica" sanificante (anche associato a gel) compare sempre nei mesi in cui si è manifestata l'epidemia in Italia; sanitizzante pure conta 4 attestazioni nel 2020 (ma la primissima è del 2013):

Arriva dalla Fra-Ber il Deotex Plus uno lo spray sanitizzante per la manutenzione dell'impianto di climatizzazione di auto e camper. [...] [C]osi alla FRA-BER hanno messo a punto un prodotto efficacissimo denominato Deotox PLUS che sanitizza e deodora gli abitacoli di auto, camper, roulotte ma naturalmente, anche di furgoni, camion, autobus, pullman, ambienti pubblici e domestici e condizionatori in generale. ([s.f.], Climatizzatore, occhio alla manutenzione, Repubblica.it, sez. Motori, 23/7/2013)

Se i quotidiani nazionali confermano la sinonimia perfetta dichiarata nei maggiori dizionari della lingua italiana, i testi legislativi mostrano invece una differenza di significato tra sanificazione e sanitizzazione, soprattutto in relazione alle procedure applicate negli ospedali e nelle strutture mediche in generale. Infatti, solo per citare un documento, nel decreto ministeriale 24 maggio 20I8, n.42 in riferimento alle competenze da raggiungere durante il percorso formativo del diploma in "Servizi per la sanità e l'assistenza sociale", si legge:

Abilità minime: applicare procedure di sanificazione e sanitizzazione. Conoscenze essenziali: caratteristiche dei detergenti, dei disinfettanti e degli asettici e modalità del loro utilizzo. (Decreto 24 maggio 2018, n. 92. Regolamento recante la disciplina dei profili di uscita degli indirizzi di studio dei percorsi di istruzione professionale, "Gazzetta Ufficiale n. 173, 27/7/2018)

Tra i dizionari si distingue il Devoto-Oli 2020, che non considera i due sostantivi sinonimi ma con sanitizzazione indica una delle fasi del processo di sanificazione. Attraverso alcune ricerche condotte su internet, si coglie che effettivamente in ambito tecnico-specialistico, soprattutto in relazione agli ospedali e alle strutture mediche, con sanitizzazione si indica la fase finale della sanificazione ovvero la fase dedicata all'eliminazione sistematica e completa di tutti gli agenti patogeni. In ambito medico, sanificazione risulta essere sinonimo di pulizia ovvero 'rimozione meccanica dello sporco da superfici e oggetti che si ottiene mediante l'uso di detergenti'. La sanitizzazione è invece la procedura messa in atto per ridurre la carica microbica su oggetti e superfici sino a un "livello di sicurezza" e si ottiene mediante l'uso prevalente di disinfettanti. Sempre in ambito medico la sanitizzazione equivale per certi aspetti (tranne per il procedimento termico) alla sterilizzazione, ovvero al 'processo termico, chimico o 
meccanico che ha lo scopo di distruggere ogni forma di vita microbica in una sostanza o in un corpo qualsiasi' (GRADIT).

Durante l'attuale emergenza si è creata una certa ambiguità tra i due termini, dovuta sostanzialmente al fatto che, ad oggi, ogni processo di pulizia vòlto all'eliminazione del virus deve contemplare le procedure di sanitizzazione (e sterilizzazione), per cui la sanificazione di un ambiente consiste nella sanitizzazione attraverso processi chimici. Sul sito dell'ISS (Istituto Superiore di Sanità), è stato pubblicato uno schema che indica la Procedura per la sanificazione (in relazione a qualsiasi tipo di ambiente, anche domestico) in cui ci si raccomanda di "utilizzare igienizzanti/sanitizzanti (es. cloro attivo, ozono) in assenza di persone". Prima della recente epidemia, con sanificazione ci si poteva riferire sostanzialmente al "mantenimento della buona qualità dell'aria" tramite procedimenti e operazioni di pulizia (si veda il Vademecum sulla Sanificazione sul sito dell'ISS). Oggi i dubbi e le incertezze circa la diffusione e permanenza del virus sulle superfici e nell'aria spinge a usare nella lingua comune i due sostantivi (e i relativi verbi) come equivalenti. Si pensi ad esempio ai comuni cartelli fuori dei supermercati in cui con "sanificare le mani" si intende l'eliminazione di germi attraverso prodotti chimici, nello specifico, gel idroalcolici.

Per quanto riguarda invece l'ambito specialistico medico, permane la differenza tra sanificazione (intesa come 'pulizia') e sanitizzazione ovvero 'fase eventuale della sanificazione, vòlta all'eliminazione di qualsiasi tipo di agente patogeno'.

Il lessico relativo all'igiene si complica ulteriormente per la presenza di derivati dell'aggettivo latino infectus (per contagiato e affetto si veda il tema di Claudio Marazzini), participio passato del verbo inficère 'mescolare, macchiare, contaminare', verbo con cui ci si riferiva "all'operazione dei tintori che mescolavano alla lana delle sostanze coloranti e secondariamente all'inquinamento delle fonti" (l'Etimologico). Da infectus derivano infettare e infezione, attestati entrambi a partire dal XIV-XV secolo. In italiano, dall'Ottocento sono attestate le parole disinfettare e disinfezione, rispettivamente dal francese désinfecter (derivato da infecter 'infettare' con l'aggiunta del prefisso dés-) e désinfection. Con disinfettare (anche in forma pronominale del tipo disinfettarsi una ferita) si intende anzitutto 'togliere le cause dell'infezione, distruggendo i germi patogeni' e con disinfezione "il complesso delle misure atte ad allontanare dall'uomo le fonti dell'infezione e a distruggere i germi patogeni (e si ha la disinfezione chimica o la disinfezione fisica a seconda che sia effettuata con disinfettanti chimici o fisici)" (GDLI). Tra i derivati di disinfettare, il più diffuso è senz'altro disinfettante (participio presente usato per lo più con valore sostantivale e aggettivale), con cui si indica comunemente il 'mezzo o la sostanza in grado di distruggere i microrganismi che provocano le infezioni' (Zingarelli 202I). Accanto a disinfezione esiste però anche il sostantivo disinfettazione, suo sinonimo, derivato di disinfettare con l'aggiunta del suffisso -zione. A differenza di disinfezione, che ha visto una mediazione francese attraverso désinfection, in questo caso si tratta di una formazione tutta italiana, che peraltro non ha avuto la stessa diffusione della precedente.

Accanto ai derivati dall'aggettivo latino infectus, ad oggi, sempre in relazione alle pratiche di igiene legate alla pandemia, si sta registrando una modesta diffusione dei termini derivati dal latino infestus 'ostile, molesto': disinfestare (derivato da infestare con l'aggiunta del prefisso privativo dis-) e disinfestazione (da infestazione), entrambi con attestazioni che risalgono ai primi anni del Novecento. Oggi i termini disinfestare e disinfestazione vengono usati quasi come sinonimi di disinfettare e disinfezione (o disinfettazione) ma anche di sanificazione, per indicare le procedure di pulizia messe in atto per eliminare la presenza del virus negli ambienti:

Prima del I4 settembre: lunghi, pesanti e costosi lavori di riadattamento delle aule scolastiche, 
disinfestazione delle aule stesse, collocazione dei nuovi banchi e delle nuove attrezzature scolastiche per adeguarle alle nuove normative anti-Covid. [...] Il I6 o I7 settembre: fine delle scuole per riadattarle a sede dei seggi elettorali, con sgombero dei banchi e attrezzature scolastiche, nuova disinfestazione (e installazione dei seggi). (Aldo Balzanelli, Scuola cenerentola tra Covid e urne, Repubblica.it, 19/8/2020)

Ora il problema è contenere il contagio. Mazzanti, dati alla mano, è sicuro che la comunità sia al sicuro. «Dal primo di maggio abbiamo avuto solo iz casi e dal 7 sono solo 8 - spiega -. Questo significa che il focolaio è circoscritto». Oggi sarà dimesso l'ultimo paziente ancora presente nel reparto di Medicina, dopodiché partiranno le procedure per la disinfestazione. (Valerio Varesi, Dopo il contagio in ospedale Budrio riscopre la paura, Repubblica.it, I6/5/2020)

In realtà (e l'uso comune ce lo suggerisce), disinfestare/disinfestazione non possono essere considerati in alcun modo sinonimi di disinfettare/disinfezione e di sanificare/sanificazione (o sanitizzare/sanitizzazione) e neanche come tali vengono registrati in tutti i dizionari della lingua italiana. Infatti con disinfestare ci si riferisce alla pratica di 'liberare da parassiti, animali dannosi o erbe nocive' (GRADIT) e dunque con disinfestazione 'il disinfestare e il suo risultato':

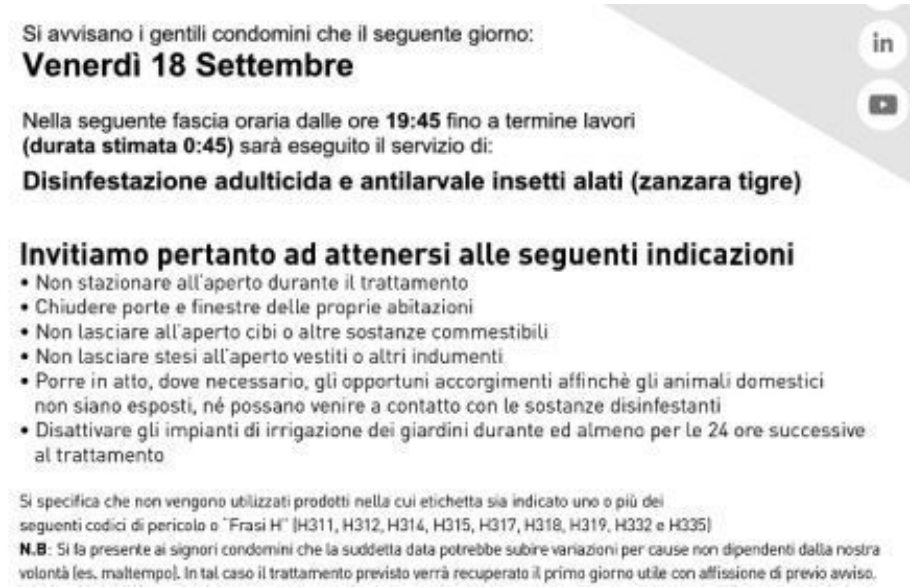

Sul sito del CEPA (Confederazione Europea dell'Industria della Disinfestazione) con sede a Bruxelles, si parla di "eliminazione degli infestanti" che prevede una fase preparatoria con "monitoraggio e controllo dei roditori; monitoraggio delle blatte e degli insetti striscianti; monitoraggio degli insetti volanti/alati". L'Associazione Nazionale delle Imprese di Disinfestazione (ANID) eroga dei corsi di formazione e tramite la descrizione delle lezioni veniamo a conoscenza di che cosa si occupa una ditta di disinfestazione: eliminazione di insetti artropodi (esapodi e aracnidi) con particolare riguardo a blatte, formiche, vespe, api, mosche, cimici dei letti, acari, zecche, zanzare, pulci ma anche ratti (in questo caso si parla piuttosto di derattizzazione), nutrie e volatili quali piccioni, storni e gabbiani ovvero ogni specie invasiva di animali. L'affiancamento dei termini disinfezione e sanificazione a quello di disinfestazione si deve, oltre a un'evidente somiglianza di questo con il primo termine, anche al fatto che il fine di tutte le procedure è l'eliminazione di un qualcosa (che sia un agente patogeno o un animale) nocivo per l'essere umano. Infatti sempre l'ANID ultimamente ha reimpostato la propria metodologia (e conseguentemente anche la terminologia), proponendo dei percorsi finalizzati alla sanificazione e disinfezione degli ambienti:

Il Presidente Marco Benedetti, inoltre, nell'augurare a tutti gli studenti impegnati in questo 2020 così particolare un buon anno scolastico, ricorda che comunque A.N.I.D persevera nel lavoro avviato di monitoraggio sulla disinfezione e sanificazione in ambito pubblico e privato, intervenendo laddove si riscontrino azioni inappropriate e arrivando anche alla diffida nei casi ritenuti pericolosi per la salute e l'ambiente. ([s.f.], Riapertura delle scuole: il punto di vista di A.N.I.D, disinfestazione.org, 2/9/2020) 
Dunque è vero che esiste qualche incertezza sull'uso di disinfestazione ma sostanzialmente in italiano il termine non può essere considerato sinonimo né di disinfezione né di sanificazione e soprattutto, per quanto riguarda i procedimenti riguardanti l'igiene personale e domestica con scopo di profilassi da infezione da nuovo coronavirus, l'uso di disinfestare e disinfestazione risulterebbe inappropriato visto che il virus non è un insetto, un animale o un'erba infestante ma un agente patogeno che, a differenza dei batteri, non è dotato di vita propria ma "parassitaria".

Un ultimo dubbio riguarda la possibile sinonimia tra sanificare/sanificazione e disinfettare/disinfezione (e/o disinfettazione). Nei testi legislativi riguardanti l'attuale emergenza epidemiologica, i sostantivi sanificazione e disinfezione vengono usati in coppia, evidentemente riferendosi a due pratiche differenti:

In considerazione del livello di esposizione al rischio di contagio da COVID-r9 connesso allo svolgimento dei compiti istituzionali delle Forze di polizia, delle Forze armate, compreso il Corpo delle Capitanerie di porto, Guardia Costiera, al fine di consentire la sanificazione e la disinfezione straordinaria degli uffici, degli ambienti e dei mezzi in uso alla medesime Forze, nonché assicurare l'adeguata dotazione di dispositivi di protezione individuale e l'idoneo equipaggiamento al relativo personale impiegato, è autorizzata la spesa complessiva di euro 23.68I.I22 per l'anno 2020, di cui euro 19,537.I22 per spese di sanificazione e disinfezione degli uffici, degli ambienti e dei mezzi [...]. (Art. 74, comma 2 del Decreto-Legge 17 marzo 2020 n. I8 convertito in Legge 24 aprile 2020, n. 27, Misure di potenziamento del Servizio sanitario nazione e di sostegno economico per le famiglie, lavoratori e imprese connesse allemergenza epidemiologica da COVID-19, "Supplemento Ordinario" n. I6 della "Gazzetta Ufficiale", 29/4/2020, n. IIO, p. 74)

Questo è uno dei tanti esempi in cui sanificazione e disinfezione compaiono insieme all'interno di un testo normativo e, stando a queste occorrenze, sembrerebbe che i due termini individuino due pratiche igieniche differenti. Se guardiamo però sempre al già citato "Schema: procedure per la sanificazione" di superfici e ambienti interni non sanitari, pubblicato sul sito dell'ISS, all'interno delle varie fasi che caratterizzano la sanificazione si legge il termine disinfezione come se il primo sostantivo fosse un iperonimo, ovvero contenesse il significato del secondo. Lo schema prevede le seguenti fasi concatenate: "devi disinfettare" > "selezionare i disinfettanti" e ancora più avanti "disinfettare periodicamente" e "disinfettare più volte al giorno". Dunque le due pratiche non possono essere considerate allo stesso livello perché prevedono due finalità differenti, sebbene la differenza non risulti ancora del tutto chiarita. L'ISS ha anche pubblicato un altro documento (ovvero il Rapporto ISS COVID-19 n. 25/2020) in cui si esplicita che "il rapporto precisa i termini usati nell'ambito della disinfezione chiarendo la differenza tra disinfettante, sanificante, igienizzante per l'ambiente e detergente":

Pertanto la sanificazione rappresenta un "complesso di procedimenti e di operazioni" che comprende attività di pulizia e/o attività di disinfezione che vanno intese "come un insieme di attività interconnesse tra di loro" quali la pulizia e la disinfezione. In alcuni casi con la sola pulizia (es. trattamenti con il calore) o con la sola disinfezione è possibile ottenere la stessa efficacia nei confronti del virus. [...]

Disinfezione. Attività che riguardano il complesso di procedimenti e operazioni atti ad abbattere la carica microbica di un ambiente superficie, strumento, ecc. Per le attività di disinfezione si utilizzano prodotti disinfettanti (biocidi o presidi medico-chirurgici) la cui efficacia nei confronti dei diversi microrganismi, come ad esempio i virus, deve essere dichiarata in etichetta sulla base delle evidenze scientifiche presentate dalle imprese stabilita a seguito dell'esame della documentazione (che include specifiche prove di efficacia) presentata al momento della richiesta di autorizzazione del prodotto. I prodotti che vantano un'azione disinfettante si configurano come PMC o come Biocidi. (Rapporto ISS COVID-19 n. 25/2020, Raccomandazioni ad interim sulla sanificazione di strutture non sanitarie nell'attuale emergenza COVID-19: superfici, ambienti interni e abbigliamento, versione del I5 maggio 2020, p. I8-19) 
In queste definizioni e in alcune precisazioni dell'ISS possiamo trovare una chiave che ci aiuta a chiarire la differenza tra sanificazione e disinfezione e perché oggi, per il nuovo coronavirus, questi due termini spesso finiscono per combaciare. Anzitutto, come si è detto all'inizio, etimologicamente la sanificazione è vòlta a rendere "sano" un ambiente, mentre la disinfezione a togliere l'"infezione". A rigor di logica in un ambiente in cui c'e stata sicuramente una persona affetta da COVID-Ig si adotteranno delle pratiche di disinfezione mentre in un ambiente in cui non si sa con certezza se siano passati individui affetti da COVID-Is si farà una sanificazione. La sanificazione non contempla per forza l'uso di prodotti disinfettanti, ovvero prodotti chimici specificamente destinati all'eliminazione di agenti patogeni: infatti, come ha precisato più volte l'ISS, la sanificazione può valersi di un qualsiasi tipo di detergente. E qui potrebbe esservi il motivo della sovrapposizione dei due termini sanificazione e disinfezione perché, nel caso del nuovo coronavirus, gli esperti specificano che per eliminare l'agente patogeno dalle superfici (qualsiasi tipo di superficie, anche dallo stesso derma umano) non siano necessari disinfettanti ma basti l'utilizzo prolungato di un detergente comune come il semplice sapone per le mani o detersivo per i pavimenti. Se facciamo una sanificazione delle mani attraverso un detergente semplice, in fin dei conti stiamo anche facendo "una disinfezione da una possibile infezione". Certo questa spiegazione non basta e risulta comunque riduttiva davanti alla grande ambiguità semantica che si è venuta a creare tra tutti i termini riguardanti l'igiene personale e domestica.

Concludendo, disinfestare/disinfestazione, sebbene usati in relazione all'eliminazione del nuovo coronavirus, non possono essere considerati termini appropriati per indicare queste pratiche di igiene degli ambienti: essi sono appropriati nei casi in cui si parli di insetti, erbe infestanti, al limite anche roditori e volatili. I termini sanificare/sanificazione si riferiscono a una procedura complessa che non contempla necessariamente l'applicazione di prodotti chimici specificamente destinati all'eliminazione di agenti patogeni. La sanificazione può essere anche una pulizia approfondita di ambienti o corpo umano tramite l'uso di semplici detergenti. Il termine sanitizzazione, spesso usato nella lingua comune come sinonimo di sanificazione, indica invece una metodologia di pulizia più specifica, caratterizzata, come nel caso della disinfezione, dall'uso di prodotti chimici creati appositamente per l'eliminazione sistematica e sicura di qualsiasi agente patogeno. Il significato tecnico specialistico appena descritto di sanitizzazione corrisponde a quello di disinfezione, con cui si indica una fase non obbligatoria del processo di sanificazione, ossia la fase che prevede l'eliminazione sistematica di agenti patogeni attraverso mezzi chimici o termici. Nell'uso attuale i termini sanificazione, sanitizzazione e disinfezione sia delle parti del corpo (in particolare le mani) sia degli ambienti sono usati molto spesso in maniera sinonimica, con netta prevalenza del primo termine sugli altri due. Vedremo se, con la progressione delle scoperte sulla trasmissione del nuovo coronavirus, i tre termini assumeranno accezioni più nettamente diversificate.

\section{Nota integrativa del $4 / 3 / 2021$ :}

Accanto ai termini ricordati va segnalata anche la moderata diffusione del termine sanifica in alternativa a sanificazione. Non era difficile prevedere la nascita e la diffusione del nome sanifica in italiano: infatti esiste un folto gruppo di nomi in -a (che alcuni studiosi chiamano "suffisso zero") che derivano da nomi in -zione, spesso e volentieri a loro volta derivanti da verbi in -ificare: ad esempio bonifica da bonificazione, classifica da classificazione, qualifica da qualificazione, verifica da verificazione. I sostantivi in -zione precedono di qualche secolo i derivati e di solito hanno lo stesso significato. Per quanto riguarda sanifica il termine non è registrato da nessun dizionario italiano e non è impiegato all'interno dei principali quotidiani nazionali ("la Repubblica", "Corriere della Sera", "la Stampa"). Sulle 
pagine in italiano di Google "la sanifica" ha I.680 risultati (ricerca del 26/2/202I) ma bisogna considerare che nella maggior parte delle occorrenze la è pronome complemento diretto femminile singolare e sanifica verbo alla terza persona singolare del presente indicativo. Se invece inseriamo "una sanifica" si hanno 458 risultati e tutti con il sostantivo di cui ci stiamo occupando. Di solito sanifica alternato a sanificazione, viene impiegato all'interno di siti che riguardano la sanificazione professionale di ambienti, superfici, aria ecc.,:

A seconda degli ambienti, dall'estensione e dagli oggetti presenti, la sanifica avviene aereosolizzando ipoclorito di sodio allo o,I\% - perossido di idrogeno allo o, $5 \%$ e, dove non sia possibile impiegare questi prodotti, sanificanti a base di Sali di quaternario al $1 \%$ o etanolo al $70 \%$. Il costo per una sanifica di questo genere può variare molto in base alle caratteristiche degli ambienti, anche perché stiamo parlando di strutture non progettate per una sanificazione costante come lo sono invece gli ospedali [...]. Dietro sopralluogo eseguiamo preventivi personalizzati e laddove ci fosse necessità, anche per pulizie ordinarie pre-sanifica e stesura del "Protocollo Sicurezza Aziendale anti contagio". (Decontaminazioni ambientali, 5 rservice.com).

Noleggiare macchinari per la sanificazione degli ambienti potrebbe essere la soluzione ideale [...]. Questo tipo di macchine per la sanifica impiega agenti biocidi in grado di eliminare batteri, virus e allergeni; nello specifico i biocidi possono essere di tipo chimico (a base di cloro, alcol, ammoniaca) o di tipo fisico (calore). [...] Le strumentazioni per la sanifica degli ambulatori medici prevedono l'impiego combinato di perossido di idrogeno e di cationi di argento garantendo una corretta disinfezione di tutte le superfici [...]. Per quanto riguarda infine i veicoli sia privati che pubblici, una corretta sanificazione è in grado di garantire un ambiente più sicuro sia per i lavoratori che per le persone, diminuendo in questo modo il rischio di contagi e diffusioni virali. Anche in questo caso una sanifica frequente e con macchinari specifici risulta necessaria. (Noleggio macchinari persanificazione ambienti, sanificazioneuffici.net)

Il fatto che sanifica si stia diffondendo in ambito specialistico in luogo del nome da cui deriva sanificazione, guardando alla maggiore diffusione di sostantivi in $-a$ a discapito di quelli da cui derivano in -zione, possiamo forse supporre che sanifica, data anche la sua brevità, possa essere destinato a imporsi in ambiti d'uso comune.

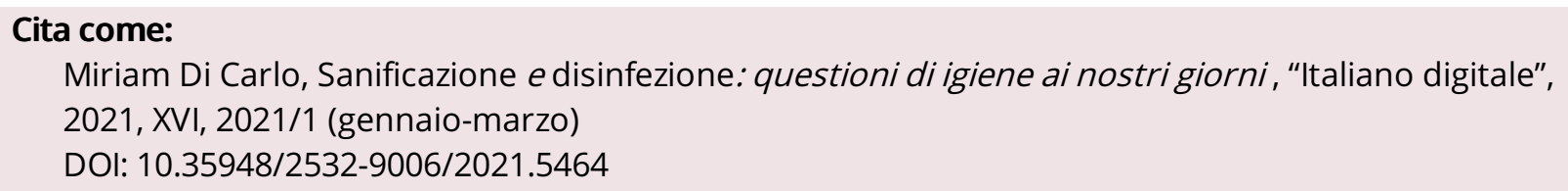

